

The Journal of Mathematics and Computer Science Vol .2 No.4 (2011) 702-716

\title{
On fuzzy homomorphisms between Hypernear-rings
}

\author{
E. Hendukolaii \\ University Callege Of Sabz, Mazandaran, Amol, Iran \\ E-mail: Edrishendoii@gmail.com
}

Received: August 2010, Revised: November 2010

Online Publication: January 2011

\begin{abstract}
We focus on the study of the structure of hypernear-rings,in this paper,we recall the basics of crisp homomorphism between hyperstructures,particularly,between hypernear-rings and, then, the notion of fuzzy homomorphism between hypernearrings is established and its main properties are analysed.
\end{abstract}

Keywords: Hypernear-rings,fuzzy homomorphisms,fuzzy ideals,fuzzy congruences.

\section{Introduction}

The study of hyperstructures started seventy-five years ago with Marty's paper [18] which firstly used a multiple-valued operator.Nowadays, the theory of hyperstructures is being thoroughly studied,focusing in particular classes such as hypergroups, hypernear-rings,semihyperrings,join spaces,etc. Mean while,fuzzy sets were introduced by Zadeh [22] and since then there has been a number of authors who started the development of fuzzy algebra. The two types of extensions presented in the previous paragraphs have started to be studied jointly,giving rise to the so-called fuzzy hyperalgebra.Several areas have benefitted from the developments in the area of hyperstructures and fuzzy set theory,in particular, artificial intelligence and soft computing: 
- hyperstructures can be used as a useful tool to modeling computing with uncertainty,in that the result of an operation is not a single value and can be considered a set of possible values;

- on the other hand,some ideas underlying fuzzy set theory form the crux on which the development of the different approaches to multiple-valued and fuzzy logics has been based.

The use of hyperstructures in conjunction with fuzzy Logic has been shown to be fruitful in some areas certainly related to artificial intelligence and soft computing, such as fuzzy logic programming with multilattices [19]. The study of hyperstructures and its (fuzzy) congruences is important both from a theoretical standpoint and for its applications in the field of logic-based approaches to uncertainty. Regarding applications, the notion of congruence is intimately related to the foundations of fuzzy reasoning and its relationships with other logics of uncertainty.Morefocused on the theoretical aspects of Computer Science, some authors $[2,20]$ have pointed out the relation be tween congruences,fuzzy automata and determinism.

The structure of the paper is the following: after stating the preliminary definitions, we recall the basics of the theory of crisp homomorphisms between hyperstructures,recalling specially the results and isomorphism theorems which relate homomorphisms ,congruences and ideals. Then,the main contribution of the paper is presented:the extension of the previous relations to the fuzzy case.

\section{Preliminaries}

Firstly,let us introduce some preliminary concepts:

Definition 2.1. A hypergroupoid is a pair $(H,+)$ consisting of a nonempty set $H$ together with a hyperoperation $+: H \times H \rightarrow 2^{H} \backslash \emptyset$.

If $A$ and $B$ are nonempty subsets of $H$, then we denote:

$$
A+B=\bigcup_{a \in A, b \in B} a+b, \quad A+x=A+\{x\}, \quad x+B=\{x\}+B .
$$

A hypergroupoid $(H,+)$ is called a semihypergroup if for all $x, y, z$ of $H$ we have $(x+y)+z=x+(y+z)$, which means that 


$$
\bigcup_{u \in x+y} u+z=\bigcup_{v \in y+z} x+v
$$

An element $e$ of $H$ is called an identity (scalar identity) of $(H,+)$ if for all $a \in H$, we have $a \in(e+a) \cap(e+a),(\{a\}=(e+a) \cap(e+a))$.

A hypergroup is a semihypergroup such that for all $x \in H$, we have $x+H=$ $H=H+x$.

A subhypergroup $(K,+)$ of $(H,+)$ is a nonempty set $K$, such that for all $k \in K$, we have $k+K=K=k+K$.

Definition 2.2. [17] A hypergroupoid $(H,+)$ is a canonical hypergroup if the following conditions hold:

(i) $(x+y)+z=x+(y+z), \quad \forall x, y, z \in H$;

(ii) $x+y=y+x$, for all $x, y \in H$;

(iii) $\exists 0 \in H$ such that $x+0=x, \quad \forall x \in H$;

(iv) $\forall x \in H, \exists ! x^{\prime} \in H$ such that $0 \in x+x^{\prime}$;

(v) $\forall x, y, z \in H$ and $z \in x+y \Longrightarrow y \in x-z$.

Definition 2.3. [9] A hypergroupoid $(H,+)$ is a quasicanonical hypergroup if the following conditions hold:

(i) $(x+y)+z=x+(y+z), \quad \forall x, y, z \in H$;

(ii) $\exists 0 \in H$ such that $x+0=x=0+x, \quad \forall x \in R$;

(iii) $\forall x \in H, \exists ! x^{\prime} \in H$ such that $0 \in\left(x+x^{\prime}\right) \cap\left(x^{\prime}+x\right)$;

(iv) $\forall x, y, z \in H$ and $z \in x+y \Longrightarrow x \in z+(-y), \quad y \in(-x)+z$.

the following equalities follow easily from the axioms:

$$
-(-x)=x \text { and }-(x+y)=-x-y .
$$

Note that in the rest of the paper we will frequently write singletons without braces.

Definition 2.4. [9] The triple $(H,+, \cdot)$ is a hypernear - ring if:

$(1)(H,+)$ is a quasicanonical hypergroup, i.e. the following axioms hold for $(R$, $+)$ :

(i) $(x+y)+z=x+(y+z), \quad \forall x, y, z \in H$;

(ii) $\exists 0 \in R$ such that $x+0=x=0+x, \quad \forall x \in H$; 
(iii) $\forall x \in H, \exists ! x^{\prime} \in H$ such that $0 \in\left(x+x^{\prime}\right) \cap\left(x^{\prime}+x\right)$;

(iv) $\forall x, y, z \in H$ and $z \in x+y \Longrightarrow x \in z+(-y), \quad y \in(-x)+z$.

$(2)(H, \cdot)$ is a semihypergroup having 0 as a right absorbing element, i.e. $0 \cdot x=0$, $\forall x \in H$

(3) $(x+y) \cdot z=x \cdot z+y \cdot z, \quad \forall x, y, z \in H$.

A subhypergroup $A \subseteq H$ is normal if we have $x+A-x \subseteq A$.

\section{On crisp homomorphisms}

We begin by discussing the different versions of the concept of homomorphism on hypegroupoids (also called multigroupoids) appearing in the literature.Some authors that deal with these and other hyperstructures use the following definitions of homomorphism[8].

Definition 3.1. [6] Let $\left(H_{1},\right)$ and $\left(H_{2},\right)$ be two hypergroupoids. A map $h: H_{1} \rightarrow H_{2}$ is said to be:

- \$Benado-homomorphism if $h(a b) \subseteq h(a) h(b)$, for all $a, b \in H_{1}$.

- $\$$ Algebraic-homomorphism if $h(a b)=h(a) h(b)$, for all $a, b \in H_{1}$.

Regarding the terminology,we depart here a bit from the usual one. The first one was the original definition by Benado [3] , which has been used in several recent papers $[8,10]$. However,it is noticeable that,finally, the authors concentrate mostly on the equality-based definition.

The terminology used in those papers is to call homo Morphism to Benado's ones and call good (or strong) Homomorphism to algebraic ones.We have adopted The term algebraic instead of good or strong because This type of homomorphism immediate allows the lifting of classical homomorphisms to the so-called powerset extension. Obviously, the advantage of using algebraic homomorphisms is that one can transfer properties from the powerset to the hypergroupoid very easily ,so that the presentation of multivalued concepts is greatly simplified. 
The term homomorphism should induce the properties of the initial hyperalgebra on the image set.It can be easily checked that this is the case for algebraichomomorphisms but,in general,it is not true for Benado-homomorphisms. The notion of homomorphism can be easily extended To the structure of hypernear-rings,since the product operation is classical.The formal definition is given below:

Definition 3.2. [1] Let $H$ and $H^{\prime}$ be two hypernear-rings. The mapping $f: H \longrightarrow$ $H^{\prime}$ is a homomorphism of hypernear-rings, if holds:

(i) $f(x+y)=f(x)+f(y)$;

(ii) $f(x \cdot y)=f(x) \cdot f(y)$;

(iii) $f(0)=0$;

for all $x, y \in H$.

Definition 3.3. [1] A normal subhypergroup A of hypergroup $(R,+)$ is

- a left hyperideal of $R$ if $x \cdot A \in A$, for all $x \in R, a \in A$;

- a right hyperideal of $R$ if $(x+A) \cdot y-x y \subseteq A$, for all $x, y \in R$;

- a bilaterally hyperideal of $R$ if $(x+A) \cdot y-x y \cup z \cdot A \subseteq A$, for all $x, y, z \in R$.

Finally,we recall below the notion of congruence relation on a hypernear-ring that we will extend.

Definition 3.4. Let $(H,+,$.$) be a hypernear-ring. A congruence on H$ is an equivalence relation $\sim$ which for all $a, b, c, d \in H$ satisfies that if $a \sim b$ and $c \sim d$ then

- for all $x \in a+c$ there exists $y \in b+d$ such that $x \sim y$,

- for all $y \in b+d$ there exists $x \in a+c$ such that $x \sim y$, and

- $a c \sim b d$.

In classical settings, it is usual to consider the kernel relation associated to a homomorphism ; this idea has been used in the framework of hyperoperations as follows:

Definition 3.5. Any hypernear-ring homomorphism $f: H \longrightarrow H^{\prime}$ defines a congruence relation, namely kernel relation $\sim_{f}$, defined as $a \sim_{f} b$ if and only if $f(a)=f(b)$. 
The relationship between the concepts of homomorphism,congruence and ideals in the framework of hyperstructures is similar to that in the classical case.

\section{Fuzzy homomorphisms on hypernear-rings}

A fuzzy relation is a mapping $\varphi$ from $H_{1} \times H_{2}$ into $[0,1]$, that is to say, any fuzzy subset of $H_{1} \times H_{2}$. The powerset extension of a fuzzy relation is defined as, $\hat{\varphi}: 2^{H_{1}} \times 2^{H_{2}} \rightarrow[0,1]$ with

$$
\hat{\varphi}(X, Y)=\left(\bigwedge_{x \in X} \bigvee_{y \in Y} \varphi(x, y)\right) \wedge\left(\bigwedge_{y \in Y} \bigvee_{x \in X} \varphi(x, y)\right)
$$

The composition of fuzzy relations $\varphi$ and $\phi$ is defined As follows:

$$
(\phi \circ \varphi)(a, c)=\bigvee_{b \in H_{2}}[\varphi(a, b) \wedge \phi(b, c)] .
$$

A fuzzy relation $\mu$ on $H \times H$ is said to be

- reflexive if $\mu(x, x)=1$, for all $x \in H$;

- symmetric if $\mu(x, y)=\mu(y, x)$,for all $x, y \in H$;

- transitive if $\mu(x, a) \wedge \mu(a, y) \leq \mu(x, y)$,for all $x, a, y \in H$.

A reflexive,symmetric and transitive fuzzy relation on $\mathrm{H}$ is called a fuzzy equivalence.A fuzzy equivalence $\mu$ on $\mathrm{H}$ is called a fuzzy equality if for any $x, y \in H$, $\mu(x, y)=1$ implies $x=y$.

Definition 4.1. A fuzzy equivalence relation $\mu$ on a hypernear-ring $(H,+,$.$) is said$ to be a fuzzy congruence relation, if for all $a, b, c, d \in H$;

- $\mu(a, b) \wedge \mu(c, d) \leq \hat{\mu}(a+c, b+d)$;

- $\mu(a, b) \wedge \mu(c, d) \leq \mu(a c, b d)$.

The fuzzification of the concept of function that we adopt has been introduced in [16],also studied in $[12,13,14]$, and more recently in [7].We will introduce the extension of the notion of perfect fuzzy function.

Definition 4.2. Let $\mu$ and $\lambda$ be two fuzzy equalities defined on the sets $H_{1}$ and $H_{2}$, respectively. $A$ partial Fuzzy function $\varphi$ from $H_{1}$ to $H_{2}$ is a mapping $\varphi$ : $H_{1} \times H_{2} \rightarrow[0,1]$ satisfying the following conditions for all $a, a^{\prime} \in H_{1}$ and $b, b^{\prime} \in H_{2}$ :

$\operatorname{ext1} \varphi(a, b) \wedge \mu\left(a, a^{\prime}\right) \leq \varphi\left(a^{\prime}, b\right)$, 
$\operatorname{ext2} \varphi(a, b) \wedge \lambda\left(b, b^{\prime}\right) \leq \varphi\left(a, b^{\prime}\right)$,

part $\varphi(a, b) \wedge \varphi\left(a, b^{\prime}\right) \leq \lambda\left(b, b^{\prime}\right)$.

If, in addition, the following condition hold:

f1 for all $a \in H_{1}$ there is $b \in H_{2}$ such that $\varphi(a, b)=1$ then we say that $\varphi$ is a perfect fuzzy function

It is not difficult to show that the element $\mathrm{b}$ in condition $(f 1)$ above is unique.As a result,every perfect fuzzy function defines a crisp mapping from $H_{1}$ to $H_{2}$ Called crisp description of $\varphi$.

Definition 4.3. Let $\left(H_{1},+,.\right)$ and $\left(H_{2},+,.\right)$ be two hypernear-rings endowed with fuzzy equalities $\mu$ and $\lambda$, respectively, such that $\lambda(a, b)=\lambda(-a,-b)$

A perfect fuzzy function $\varphi \in[0,1]^{H_{1} \times H_{2}}$ is said to be a fuzzy homomorphism if for all $a_{1}, a_{2} \in H_{1}$ and $b_{1}, b_{2} \in H_{2}$ the following conditions hold:

- $\varphi\left(a_{1}, b_{1}\right) \wedge \varphi\left(a_{2}, b_{2}\right) \leq \hat{\varphi}\left(a_{1}+a_{2}, b_{1}+b_{2}\right)$

- $\varphi\left(a_{1}, b_{1}\right) \wedge \varphi\left(b_{1}, b_{2}\right) \leq \varphi\left(a_{1} a_{2}, b_{1} b_{2}\right)$

- $\varphi(0,0)=1$

Moreover, $\varphi$ is said to be complete if the following conditions hold:

1. if $\bigvee_{y \in Y} \varphi(a, y)=1$, then there exists $y \in H_{2}$ such that $\varphi(a, y)=1$,

2. if $\bigvee_{x \in X} \varphi(x, b)=1$, then there exists $x \in H_{1}$ such that $\varphi(x, b)=1$.

Remark:Hereafter,unless stated otherwise, we will always consider that we are working with a complete fuzzy homomorphism $\varphi$ between hypernear-rings $H_{1}$ and $H_{2}$ And fuzzy equalities $\mu$ and $\lambda$,respectively.

Proposition 4.4. Given $\varphi$ between $H_{1}$ and $H_{2}$, the crisp description $f$ of $\varphi$ is a hypernear-ring homomorphism.

The notion of fuzzy homomorphism between hypernear-rings behaves properly with respect to the composition of fuzzy relations, in that the composition of fuzzy homomorphisms is a fuzzy homomorphism.Furthermore,the composition is associative and there exists an identity for this composition. As a result, the class of hypernear-rings together with the fuzzy homomorphisms between them forms a category. 
Let us concentrate now on the relationship between fuzzy homomorphism and congruences.

Definition 4.5. the fuzzy kernel relation induced by $\varphi$ in $H_{1}$ is defined as $\mu_{\varphi}\left(a, a^{\prime}\right)=$ $\varphi\left(a, f\left(a^{\prime}\right)\right)$.

We adopt here the term kernel as an extension of the crisp case because of the inequality

$$
\varphi(a, b) \wedge \varphi\left(a^{\prime}, b\right) \leq \mu_{\varphi}\left(a, a^{\prime}\right) .
$$

Moreover, in [7], the authers prove the following result.

Proposition 4.6. Let $\varphi$ be a perfect fuzzy function from $H_{1}$ to $H_{2}$. For all $a, a^{\prime} \in$ $H_{1}$, we have:

$$
\mu_{\varphi}\left(a, a^{\prime}\right)=\bigvee_{b \in H_{2}} \varphi(a, b) \wedge \varphi\left(a^{\prime}, b\right)
$$

In the case of fuzzy homomorphisms between hypernear-rings, we prove that this fuzzy equivalence relations also a fuzzy congruence on the initial hypernear-ring.

Theorem 4.7. Consider $\varphi$ between $H_{1}$ and $H_{2}$. The fuzzy kernel relation $\mu_{\varphi}$ is a fuzzy congruence relation Which includes the fuzzy equality $\mu$ in $H_{1}$.

Proof. Let us see that $\mu_{\varphi}$ is compatible with the sum operation,then we have:

$$
\begin{aligned}
& \hat{\mu_{\varphi}}\left(a_{1}+a_{3}, a_{2}+a_{4}\right)= \\
& =\left(\bigwedge_{a \in a_{1}+a_{3}} \bigvee_{a^{\prime} \in a_{2}+a_{4}}\left[\mu_{\varphi}\left(a, a^{\prime}\right)\right]\right) \wedge\left(\bigwedge_{a^{\prime} \in a_{2}+a_{4}} \bigvee_{a \in a_{1}+a_{3}}\left[\mu_{\varphi}\left(a, a^{\prime}\right)\right]\right) \\
& =\hat{\mu_{\varphi}}\left(a_{1}+a_{3}, f\left(a_{2}+a_{4}\right)\right) \quad \text { as } \quad \mu_{\varphi}\left(a, a^{\prime}\right)=\varphi\left(a, f\left(a^{\prime}\right)\right) \\
& =\hat{\mu_{\varphi}}\left(a_{1}+a_{3}, f\left(a_{2}\right)+f\left(a_{4}\right)\right) \\
& \geq \varphi\left(a_{1}, f\left(a_{2}\right)\right) \wedge \varphi\left(a_{3}, f\left(a_{4}\right)\right) \\
& =\mu_{\varphi}\left(a_{1}, a_{2}\right) \wedge \mu_{\varphi}\left(a_{3}, a_{4}\right)
\end{aligned}
$$

The compatibility with the multiplication follows from

$$
\begin{aligned}
\mu_{\varphi}\left(a_{1} a_{3}, a_{2} a_{4}\right) & =\varphi\left(a_{1} a_{3}, f\left(a_{2} a_{4}\right)\right) \\
& =\varphi\left(a_{1} a_{3}, f\left(a_{2}\right) f\left(a_{4}\right)\right) \quad \text { by Prop. } 12 \\
& \geq \varphi\left(a_{1}, f\left(a_{2}\right) \wedge \varphi\left(a_{3}, f\left(a_{4}\right)\right.\right. \\
& =\mu_{\varphi}\left(a_{1}, a_{2}\right) \wedge \mu_{\varphi}\left(a_{3}, a_{4}\right)
\end{aligned}
$$

Now,let us show that $\mu \leq \mu_{\varphi}$ : 


\section{E. Hendukolaii/ TJMCS Vol .2 No.4 (2011) 702-716}

$$
\begin{aligned}
\mu\left(a, a^{\prime}\right) & =\mu\left(a, a^{\prime}\right) \wedge \varphi\left(a^{\prime}, f\left(a^{\prime}\right)\right) \\
& \leq \varphi\left(a, f\left(a^{\prime}\right)\right)=\mu_{\varphi}\left(a, a^{\prime}\right) \quad \text { by } \quad(\operatorname{ext} 1)
\end{aligned}
$$

In the rest of this section we will show the canonical Decomposition theorem for a complete fuzzy homomorphism and a fuzzy congruence relation.For suitable extensions on the notions of injectivity and surjectivity we will rely on the definitions given in [12].

Definition 4.8. A perfect fuzzy function $\varphi \in[0,1]^{H_{1} \times H_{2}}$ is said to be:

- surjective if for all $b \in H_{2}$ there exists $a \in H_{1}$ such that $\varphi(a, b)=1$.

- injective if $\varphi(a, b) \wedge \varphi\left(a^{\prime}, b\right) \leq \mu\left(a, a^{\prime}\right)$ for all $a, a^{\prime} \in H_{1}$ and $b \in H_{2}$.

- bijective if it is injective and surjective.

The image set is $\operatorname{Im} \varphi=\left\{b \in H_{2} \mid\right.$ thereexistsa $\in H_{1}$ with $\left.\varphi(a, b)=1\right\}$.

In order to define the different homomorphisms involved in the decomposition theorem, we have to introduce the quotient set associated to a fuzzy equivalence relation.

Definition 4.9. Let $(H,+,$.$) be a hypernear-ring and \mu$ be a fuzzy equivalence relation in $H$. An equivalence Class of an element $a \in H$ is defined as:

$$
\mu(a) \in[0,1]^{H} \text { with } \mu(a)\left(a^{\prime}\right)=\mu\left(a, a^{\prime}\right)
$$

The quotient set is defined as $H / \mu=\{\mu(a) \mid a \in H\}$ And a fuzzy equality $\bar{\mu}$ can be defined in $H / \mu$ as:

$$
\bar{\mu}\left(\mu(a), \mu\left(a^{\prime}\right)\right)=\mu\left(a, a^{\prime}\right) .
$$

The fuzzy projection $\tau$ from $H$ to $H / \mu$ is defined as $\tau\left(a, \mu\left(a^{\prime}\right)\right)=\mu\left(a, a^{\prime}\right)$.

Proposition 4.10. Let $(H,+,$.$) be a hypernear-ring and \mu$ be a fuzzy equality in $H$ and $\mu_{H}$ be a fuzzy congruence relation in $H$ that includes $\mu$. The fuzzy projection $\tau$ from $\mathrm{H}$ to $H / \mu_{H}$ is a surjective fuzzy homomorphism where the hyperoperations in $H / \mu_{H}$ are given by

$$
\begin{aligned}
& \mu_{H}\left(a_{1}\right)+\mu_{H}\left(a_{2}\right)=\left\{\mu_{H}(c) \mid c \in a_{1}+a_{2}\right\} \\
& \mu_{H}\left(a_{1}\right) \cdot \mu_{H}\left(a_{2}\right)=\left\{\mu_{H}(c) \mid c \in a_{1} a_{2}\right\}
\end{aligned}
$$

The zero element is $\mu_{H}(0)$ and the fuzzy equality is $\bar{\mu}_{H}$. 
Remark. In order to prove that the canonical inclusion from the image of a homomorphism is an injective fuzzy homomorphism,were call the following result from [12]:given $\varphi$ between $H_{1}$ and $H_{2}$, there exists a unique crisp function $h$ such that $\varphi(a, b)=\lambda(h(a), b)$. This $h$ actually coincides with the crisp description $f$ Of $\varphi$, which satisfies $\varphi(a, f(a))=1$.

Lemma 4.11. Given $\varphi$ between $H_{1}$ and $H_{2}$, then the inclusion $\iota$ from $\operatorname{Im} \varphi$ to $H_{2}$ defined as $\iota\left(b, b^{\prime}\right)=\lambda\left(b, b^{\prime}\right)$ is an injective fuzzy homomorphism.

Theorem 4.12. Any complete fuzzy homomorphism $\varphi$ from $\mathrm{H}_{1}$ to $\mathrm{H}_{2}$ can be canonically decomposed as $\varphi=\iota \circ \bar{\varphi} \circ \tau$ where $\tau$ is the fuzzy projection from $H_{1}$ to $H_{1} / \mu \varphi$, and $\iota$ is the inclusion from $\operatorname{Im} \varphi$ to $H_{2}$, and $\bar{\varphi}$ Is the isomorphism from $H_{1} / \mu \varphi$ to Im $\varphi$ defined as $\bar{\varphi}(\mu \varphi(a), b)=\varphi(a, b)$, and the operations and the fuzzy equality in Im $\varphi$ being the corresponding restrictions of those in $\mathrm{H}_{2}$.

Proof. We will prove $(e x t 1),(i n j)$ and $(s u r j)$ for $\bar{\varphi}$ Since the rest of properties are straightforward:

$$
\begin{aligned}
\text { (ext1) } & \bar{\varphi}\left(\mu_{\varphi}(a), b\right) \wedge \bar{\mu}_{\varphi}\left(\mu_{\varphi}(a), \mu_{\varphi}\left(a^{\prime}\right)\right) \\
= & \varphi(a, b) \wedge \mu_{\varphi}\left(a, a^{\prime}\right) \\
= & \varphi(a, b) \wedge \varphi\left(a, f\left(a^{\prime}\right)\right) \\
= & \varphi(a, b) \wedge \varphi\left(a, f\left(a^{\prime}\right)\right) \wedge \varphi\left(a^{\prime}, f\left(a^{\prime}\right)\right) \\
\leq & \lambda\left(b, f\left(a^{\prime}\right)\right) \wedge \varphi\left(a^{\prime}, f\left(a^{\prime}\right)\right) \\
\leq & \varphi\left(a^{\prime}, b\right)=\bar{\varphi}\left(\mu_{\varphi}\left(a^{\prime}\right), b\right) \\
(\text { inj }) \quad & \left(\mu_{\varphi}(a), b\right) \wedge \bar{\varphi}\left(\mu_{\varphi}\left(a^{\prime}\right), b\right) \\
= & \varphi(a, b) \wedge \varphi\left(a^{\prime}, b\right) \\
\leq & \mu_{\varphi}\left(a, a^{\prime}\right)=\bar{\mu}_{\varphi}\left(\mu_{\varphi}(a), \mu_{\varphi}\left(a^{\prime}\right)\right)
\end{aligned}
$$

(surj) For all $b \in \operatorname{Im} \varphi$ there exists $a \in H_{1}$ such that $\varphi(a, b)=1$ and then $\bar{\varphi}\left(\mu_{\varphi}(a), b\right)=1$

Now,let us check that $\varphi=\iota \circ \bar{\varphi} \circ \tau$ :

$$
\begin{aligned}
(\varphi= & \iota \circ \bar{\varphi} \circ \tau)(a, b)= \\
& \bigvee_{\mu_{\varphi}\left(a^{\prime}\right) \in H_{1} / \mu \varphi, b^{\prime} \in i m \varphi}\left[\tau\left(a, \mu_{\varphi}\left(a^{\prime}\right) \wedge \bar{\varphi}\left(\mu_{\varphi}\left(a^{\prime}\right), b^{\prime}\right) \wedge \iota\left(b^{\prime}, b\right)\right)\right] \\
& =\bigvee_{a^{\prime} \in H_{1}, b^{\prime} \in \operatorname{im\varphi } \varphi}\left[\mu_{\varphi}\left(a, a^{\prime}\right) \wedge \varphi\left(a^{\prime}, b^{\prime}\right) \wedge \lambda\left(b^{\prime}, b\right)\right]
\end{aligned}
$$




$$
\begin{aligned}
& \leq \bigvee_{a^{\prime} \in H_{1}}\left[\mu_{\varphi}\left(a, a^{\prime}\right) \wedge \varphi\left(a^{\prime}, b\right)\right] \\
& =\bigvee_{a^{\prime} \in H_{1}}\left[\varphi\left(a, f\left(a^{\prime}\right) \wedge \varphi\left(a^{\prime}, b\right)\right]\right. \\
& =\bigvee_{a^{\prime} \in H_{1}}\left[\varphi\left(a, f\left(a^{\prime}\right) \wedge \varphi\left(a^{\prime}, f\left(a^{\prime}\right)\right) \wedge \varphi\left(a^{\prime}, b\right)\right]\right. \\
& \leq \bigvee_{a^{\prime} \in H_{1}}\left[\varphi\left(a, f\left(a^{\prime}\right) \wedge \lambda\left(f\left(a^{\prime}\right), b\right)\right]\right. \\
& \leq \varphi(a, b) .
\end{aligned}
$$

Conversely, $\varphi(a, b)=\lambda(f(a), b)=\tau\left(a, \mu_{\varphi}(a)\right) \wedge \bar{\varphi}\left(\mu_{\varphi}(a), f(a)\right) \wedge \lambda(f(a), b) \leq(\iota \circ \bar{\varphi} \circ$ $\tau)(a, b)$.

\section{$5 \quad$ Fuzzy hyperideals and homomorphisms}

This section is devoted to the fuzzy extension of the Classical relation between crisp ideals and homomorphisms. First of all,we adopt the definition of fuzzy hyperideal defined. Nevertheless, the superfluous conditions have been removed.

Definition 5.1. Let $(H,+,$.$) be a hypernear-ring. A fuzzy subset \rho$ of $H$ is a fuzzy hyperideal if it satisfies, for All $a, b \in H$ we have:

(1) $\rho(a) \wedge \rho(b) \leq \rho(x)$ for all $x \in a-b$,

(2) $\rho(a) \vee \rho(b) \leq \rho(a b)$.

The kernel relation in a hypernear-ring can be expressed in terms of the kernel of the corresponding homomorphism. In this section we prove that the same occurs in the fuzzy case, due to the convenient properties including in our definition of fuzzy homomorphism.

Let $\left(H_{1},+,.\right)$ and $\left(H_{2},+,.\right)$ be two hypernear-rings endowed with fuzzy equalities $\mu$ and $\lambda$,respectively, such that $\lambda(a, b)=\lambda(-a,-b)$.

Let us consider now the fuzzy kernel relation induced By $\varphi$ in $H_{1}, \mu_{\varphi} \in[0,1]^{H_{1} \times H_{1}}$ , defined as $\mu_{\varphi}\left(a, a^{\prime}\right)=\varphi\left(a, f\left(a^{\prime}\right)\right)$, where $h$ is the crisp description of $\varphi$.

Note that $\mu_{\varphi}(0) \in[0,1]^{H_{1}}$ is a fuzzy subset of $H_{1}$ and,

$$
\mu_{\varphi}(0)(a)=\mu_{\varphi}(0, a)=\varphi(0, f(a))=\lambda(0, f(a))=\lambda(f(a), 0)=\varphi(a, 0)
$$

Theorem 5.2. Consider $\varphi$ between $H_{1}$ and $H_{2}$ and the fuzzy kernel relation $\mu_{\varphi}$. Then $\mu_{\varphi}(0)$ is a fuzzy hyperideal. 
Proof. Firstly,observe that $\varphi(a, b)=\lambda(f(a), b)=\lambda(-f(a),-b)=\lambda(f(-a),-b)=$ $\varphi(-a,-b)$ for all $a \in H_{1}$ and $b \in H_{2}$. On the other hand,

$$
\begin{aligned}
\mu_{\varphi}(0)(a) \wedge \mu_{\varphi}(0)(b)= & \\
& \varphi(0, f(a)) \wedge \varphi(0, f(b)) \\
& =\varphi(0, f(a)) \wedge \varphi(0,-f(b)) \\
& \leq \hat{\varphi}(0, f(a)-f(b)) \\
& =\hat{\varphi}(0, f(a-b)) \\
& =\bigwedge_{y \in f(a-b)} \varphi(0, y) \\
& =\bigwedge_{x \in a-b} \varphi(0, f(x)) \\
& =\bigwedge_{x \in a-b} \mu_{\varphi}(0)(x)
\end{aligned}
$$

So $\mu_{\varphi}(0)(a) \wedge \mu_{\varphi}(0)(b) \leq \mu_{\varphi}(0)(x)$ for al $x \in a-b$.

Related to the multiplication,

$$
\mu_{\varphi}(0)(a)=\mu_{\varphi}(0, a)=\mu_{\varphi}(0, a) \wedge \mu_{\varphi}(b, b) \leq \mu_{\varphi}(0, a . b)=\mu_{\varphi}(0)(a . b) .
$$

Once proven that the kernel of a fuzzy homomorphism between hypernear-rings is a fuzzy hyperideal,we consider whether the existing relationship between the fuzzy hyperideal and the congruence defined by the fuzzy homomorphisms is what one could expect.

In the crisp case,given an ideal $I$, a congruence is defined [11] by;

$a \sim b \quad(\bmod I)$ if and only if $(a-b) \cap I \neq \emptyset$

The natural form of fuzzifying this construction would be the following:if $\rho$ is a fuzzy hyperideal of $H$, the fuzzy relation $\mu_{\rho} \in[0,1]^{H \times H}$ should be;

$$
\mu_{\rho}\left(a, a^{\prime}\right)=\bigvee_{x \in a-a^{\prime}} \rho(x)
$$

In a nutshell,the fuzzy subset $\mu_{\varphi}(0)$ plays the role of the kernel of the homomorphism in the crisp case.

Theorem 5.3. Consider $\varphi$ between $H_{1}$ and $H_{2}$ and the fuzzy kernel relation $\mu_{\varphi}$. Then

$$
\mu_{\varphi}\left(a, a^{\prime}\right)=\bigvee_{x \in a-a^{\prime}} \mu_{\varphi}(0)(x)
$$

Proof. Firstly we prove $\mu_{\varphi}\left(a, a^{\prime}\right) \leq \bigvee_{x \in a-a^{\prime}} \mu_{\varphi}(0)(x)$.

From Proposition 4.6 , 


$$
\mu_{\varphi}\left(a, a^{\prime}\right)=\varphi\left(a, f\left(a^{\prime}\right)\right)=\bigvee_{b \in H_{2}} \varphi(a, b) \wedge \varphi\left(a^{\prime}, b\right)
$$

On the other hand, for all $b \in H_{2}$, we have:

$$
\begin{aligned}
\varphi(a, b) \wedge \varphi\left(a^{\prime}, b\right) & \leq \hat{\varphi}\left(a-a^{\prime}, b-b\right) \\
& =\left[\bigwedge_{x \in a-a^{\prime}} \bigvee_{y \in b-b} \varphi(x, y)\right] \wedge\left[\bigwedge_{y \in b-b} \bigvee_{x \in a-a^{\prime}} \varphi(x, y)\right] \\
& \left.\leq \bigwedge_{y \in b-b} \bigvee_{x \in a-a^{\prime}} \varphi(x, y)\right] \\
& \leq \bigvee_{x \in a-a^{\prime}} \varphi(x, 0) \\
& =\bigvee_{x \in a-a^{\prime}} \mu_{\varphi}(0)(x)
\end{aligned}
$$

Thus, $\bigvee_{b \in H_{2}} \varphi(a, b) \wedge \varphi\left(a^{\prime}, b\right) \leq \bigvee_{x \in a-a^{\prime}} \mu_{\varphi}(0)(x)$ and by (1),one obtains the inequality required.Now, we check that $\bigvee_{x \in a-a^{\prime}} \varphi(x, 0) \leq \mu_{\varphi}\left(a, a^{\prime}\right)$. It suffices to prove that $\mu_{\varphi}\left(a, a^{\prime}\right)$ is an upper bound.

$\mu_{\varphi}(0)(x)=\mu_{\varphi}(x, 0)=\mu_{\varphi}(x, 0) \wedge \mu_{\varphi}\left(a^{\prime}, a^{\prime}\right) \leq \hat{\mu}_{\varphi}\left(x+a^{\prime}, a^{\prime}\right)=\bigwedge_{z \in x+a^{\prime}} \mu_{\varphi}\left(z, a^{\prime}\right) \leq$ $\mu_{\varphi}\left(a, a^{\prime}\right)$.

\section{References}

[1] R. Ameri, E. Hendoukolaii, Fuzzy Hypernear-rings, Ital. J. Pure Appl. Math. to appear

[2] R. Belohlavek. Determinism and fuzzy automata. In Formation Sciences, 143: 205-209,2002.

[3] M. Benado. Les ensembles partiellement ordonnes et Le theorem derafinement de Schreier. I. Cehoslovack. Mat. Z., 4(79): 105-129, 1954.

[4] I. P. Cabrera, P. Cordero, G. Gutierrez, J. Martinez, And M. Ojeda- Aciego. Fuzzy congruence relations on nd-groupoids. Intl J on Computer Mathematics 86: 1684-1695, 2009.

[5] I.P. Cabrera, P. Cordero, G. Gutierrez, J. Martinez, And M. Ojeda- Aciego. Congruence relations on some hyperstructures. Annals of Math. And Artif. Intell. Article in press (doi: 10.1007/s10472-009-9146-5). 
[6] I.P. Cabrera, P. Cordero, G. Gutierrez, J. Martinez, And M. Ojeda- Aciego. On fuzzy homomorphism between hyperrings. spanish Science Ministry. TIN200615455- C03-01.

[7] M. Ciric, J. Ignjatovic, and S. Bogdanovic. Uniform Fuzzy relations and fuzzy functions. Fuzzy Sets and Systems,160 (8) :1054-1081, 2009.

[8] P. Corsini. A new connection between hypergroups and fuzzy sets. Southeast Asian Bull. Math., 27(2):221-229, 2003.

[9] V. Dasic, Hypernear-rings, in: Proc. Fourth Int. Congress on AHA (1990), World Scientific, 1991, pp. 75-85.

[10] B. Davvaz. Some results on congruences on semihypergroups. Bulletin of the Malaysian Mathematical Sciences Society. Second Series,23(1):53-58, 2000.

[11] B. Davvaz. Isomorphism theorems of hyperrings. Indian J. Pure Applied Mathematics,35(3):321-331, 2004.

[12] M. Demirci.Fuzzy functions and their applications. J. Math. Anal. Appl, 252:495-517, 2000.

[13] M. Demirci. Gradation of being fuzzy function. Fuzzy Sets and Systems,119(3):383-392, 2001.

[14] M. Demirci. Constructions of fuzzy functions based on fuzzy equalities. Soft Computing, 7(3):199-207, 2003.

[15] V.M. Gontineac, On hypernear-rings and H-hypergroups, in: Proc. Fifth Int. Congress on AHA (1993), Hadronic Press Inc., USA, (1994), 171-179.

[16] F. Klawonn. Fuzzy points, fuzzy relations and fuzzy function. In V. NovakandI. Perfilieva, editors, Discovering world with Fuzzy Logic, pages 431-453. PhysicaVerlag, 2000.

[17] M. Krasner. A class of hyperrings and hyperfields. International Journal of Mathematics and Mathematical Sciences, 6(2):307-311,1983. 
[18] F. Marty, Sur une généralisation de la notion de group, in: 8th Congress Math. Scandinaves, Stockholm, 1934, pp. 4549.

[19] J. Medina and M. Ojeda-Aciegoand J. Ruiz-Calvino. Fuzzy logic programming via multilattices. Fuzzy Sets And Systems. N. 158,Vol.6, Pag. 674-688, 2007.

[20] T.Petkovic. Congruences and homomorphisms of Fuzzy automata. Fuzzy Sets and Systems,157:444- 458, 2006.

[21] T. Vougiouklis, Hyperstructures and Their Representations, Hadronic Press Inc., Palm Harber, 1994, p. 115.

[22] L. A. Zadeh. Fuzzy sets. Information and Control, 8:338-353, 1965.

[23] J. Zhan, B. Davvaz, and K.P.Shum. A new view of Fuzzy hypernear-rings. Inf. Sci.,178(2):425-438, 2008. 